Immunisation

\title{
Critical evaluation of smallpox vaccination for laboratory workers
}

\section{S N Isaacs}

\section{An alternative view}

A witnessed by the eradication of naturally occurring smallpox and near eradication of polio, vaccination against infectious diseases has been a true success story of medical science. The use of safe and efficacious vaccines to control highly contagious and morbid diseases extends to the occupational setting as well. When safe and effective vaccines are available, using them in an occupational setting to protect workers from diseases to which they may be exposed is obvious. This is clearly the case for protection of healthcare workers against measles, mumps, rubella, varicella, and hepatitis B. Animal handlers should also be vaccinated against rabies. These vaccines all target viral infections that are easily transmitted and/or result in serious illnesses. Similarly, if a laboratory worker handles a potential pathogen for which a safe and effective vaccine exists, in most cases vaccination is warranted.

Recently, the Advisory Committee on Immunization Practices (ACIP) updated their recommendations for the use of vaccinia vaccine. ${ }^{1}$ The revised ACIP recommendations address the nonemergency use of vaccinia virus vaccine in laboratory, animal care, and healthcare workers with potential occupational exposure to vaccinia virus. The ACIP continues to strongly recommend the vaccination of laboratory workers and animal handlers who may come in contact with non-attenuated strains of vaccinia virus, or with other orthopoxviruses that can infect humans, such as monkeypox. Of note, there are a few major changes and an addition to the prior recommendations given a decade earlier.

The revised ACIP guidelines now no longer recommend vaccinia vaccine to researchers working with specific highly attenuated or non-replicating poxviruses. Such attenuated and avian poxviruses do not replicate in mammalian cells and are avirulent in both normal and immunocompromised animals, thus negating the need for protective vaccination. The new guidelines also no longer recommend routine vaccination of healthcare workers who handle experimental recombinant vaccinia virus vaccines during clinical studies. This change was instituted because it was felt that viral transmission from patient to provider should not occur with proper infection control precautions. Finally, the ACIP added new recommendations regarding the use of the vaccine in the event of smallpox bioterrorism. Obviously, the discussion that follows does not apply to the use of the vaccine to prevent smallpox, which is a highly contagious disease with excessive mortality.

\section{"Vaccinia vaccination is by definition an infection"}

The ACIP continues to recommend vaccination for individuals who work with vaccinia viruses in research facilities. Unfortunately, the ACIP did not acknowledge or discuss the alternative perspective that routine vaccination of lab workers who use vaccinia virus (or animal care givers who may come in contact with infected animals) might be more problematic than working safely with the virus using good microbiological techniques without vaccination. ${ }^{2}$ Indeed, vaccinia vaccination is by definition an infection and thus the ACIP is recommending universal "controlled" vaccinia virus infection to prevent rare accidental vaccinia virus infections. We must recognise that there is a small, yet very real risk of serious complications, and a higher frequency of less serious but disturbing sequelae from a "controlled" vaccinia vaccine associated infection.

Over a decade ago, two advisory committees in the UK published their recommendations for vaccination of laboratory workers handling vaccinia virus. ${ }^{4}$ Their conclusions were quite different from those reached by the ACIP. The UK committees concluded that the risk of complications and serious side effects outweigh the benefit of routine vaccination among workers handling vaccinia virus, and vaccination should be considered only in special circumstances. It is disappointing that this viewpoint was not addressed in the recent ACIP publication. This issue is even more important now because there is a growing population of workers born after 1972 who have never been vaccinated, and so would be receiving vaccine for the first time. Such primary vaccinees are at a 10 - to 20 -fold greater risk for complications from vaccination than was seen during prior times when most occupational smallpox vaccinations represented a booster dose. ${ }^{5}$ In fact, such surveillance studies have shown that primary vaccination of adults results in more overall complications than in children over the age of 1. Some even argue that serious complications such as neurological sequelae may be especially more frequent in adults receiving vaccine for the first time than in children. ${ }^{6}$ However, even less serious complications such as generalised vaccinia are important to consider, because such undesirable side effects might not be acceptable in otherwise healthy laboratory personnel. It is also known that quantification of the less serious complications from the vaccine during smallpox eradication is known to be underestimated. ${ }^{5}$ I do not believe the ACIP weighed such concerns adequately when formulating their recommendations. While the ACIP cited the very low rate of serious complications in adults reported from recent vaccination experience by the Israeli army, they failed to discuss the high rate of less serious complications that was likely a result of an increased percentage of primary vaccinees. $^{7}$

\section{"The decision to vaccinate should be an individualised one"}

As written, the unambiguous tone and dogmatic conclusion of the ACIP to uniformly recommend routine vaccination for workers who handle vaccinia virus obscures the fact that the decision to vaccinate is not clear cut. This creates a troubling situation because most institutions in the United States strictly follow the ACIP recommendations and, in some cases, make vaccination mandatory to work with vaccinia virus. The risks and benefits of the vaccine have to be weighed against the exact duties of the worker. The risk-benefit ratio of vaccination might be tipped towards recommending vaccinia vaccination of workers who prepare large volumes of high titre vaccinia virus, work with recombinant vaccinia viruses that might have enhanced virulence or that express harmful proteins, or inject high titre vaccinia virus into animals. With that said, the decision to vaccinate should be an individualised one: one that is made with consideration of the particular situation, recognition of the uncertainties involved, and that incorporates the individual worker's perspective as well.

Given fears about smallpox bioterrorism, some workers who are eligible to 
receive the vaccine because of occupational considerations might believe that receiving it for protection against bioterrorism is an additional benefit. Despite recent world events, no naturally occurring smallpox has occurred in nearly a quarter of a century and the risk of bioterrorism with smallpox remains purely theoretical. Thus this perceived benefit must be kept in perspective and is likely quite small.

Accidental infections with vaccinia virus in the workplace can be avoided with good microbiological techniques. Furthermore, the hazard for a worker is a personal one and, in contrast to many other infectious diseases, is not a significant risk for co-workers, family, or the general public. The risk-benefit ratio of the vaccinia virus vaccine was certainly acceptable for the prevention of smallpox. I do not think the data support routine vaccination of all people who work with vaccinia virus. I believe a more appropriate strategy, especially when expected complication rates from the vaccine will be greater because of the high percentage of primary vaccinees, is to individualise the decision in the setting of knowledgeable counselling. ${ }^{8}$

Occup Environ Med 2002;59:573-574

\section{Authors' affiliations}

S N Isaacs, University of Pennsylvania, Division of Infectious Diseases, Philadelphia,

Pennsylvania, USA

Correspondence to: Dr S Isaacs, Assistant Professor of Medicine, University of Pennsylvania, Division of Infectious Diseases, 502 Johnson Pavilion, Philadelphia, PA 19104-6073, USA;

isaacs@mail.med.upenn.edu

\section{REFERENCES}

1 Vaccinia (smallpox) vaccine recommendations of the Advisory Committee on Immunization
Practices (ACIP), 2001. MMWR Morb Mortal Wkly Rep 2001;50:1-25.

2 Wenzel RP, Nettleman MD. Smallpox vaccination for investigators using vaccinia recombinants. Lancet 1989:2:630-1.

3 Baxby D. Indications for smallpox vaccination: policies still differ. Vaccine 1993;11:395-6.

4 Advisory Committee on Dangerous Pathogens and Advisory Committee on Genetic Modification. Vaccination of laboratory workers handling vaccinia and related poxviruses infectious for humans. London: HMSO Publications, 1990:1-16.

5 Lane JM, Ruben FL, Neff JM, et al. Complications of smallpox vaccination, 1968 National surveillance in the United States. $N$ Engl J Med 1969;281:1201-8.

6 Gurvich EB. The age-dependent risk of postvaccination complications in vaccinees with smallpox vaccine. Vaccine 1992;10:96-7.

7 Haim M, Gdalevich M, Mimouni D, et al. Adverse reactions to smallpox vaccine: the Israel defense force experience, 1991 to 1996. A comparison with previous surveys. Mil Med 2000; 165:287-9.

8 Williams NR, Cooper BM. Counselling of workers handling vaccinia virus. Occup Med (Oxf) 1993;43: 125-7.

\section{OEM web submission and review system}

I am pleased to inform authors and reviewers of the new online submission and review system at OEM. Developed by Highwire Press (CA, USA), Bench $>$ Press is a fully integrated electronic system which uses the internet to allow rapid and efficient submission of manuscripts. It also allows the peer review process to be conducted entirely online. The main aim is to speed up the frequently frustrating progress from submission to publication.

Authors can submit their manuscript in any standard word processing software. Standard graphic formats acceptaed include: .jpg, .tiff, .gif, eps, etc. (Please note: multi page powerpoint files are not accepted by the BM Publishing Group.) The text and graphic files are automatically converted to PDF for ease of distribution and reviewing purposes. Authors are asked to approve their submission before it formally enters the reviewing process. On approval, the submission is passed to the editor and/or reviewers via the web. All transactions are secure.

To access the system click on "SUBMIT YOUR MANUSCRIPT HERE" on the OEM homepage: http://www.occenvmed.com, or you can access the submission site directly at http://submitoem.bmijournals.com.

We are very excited with this new development and would encourage authors and reviewers to use the system where possible. It really is simple to use and should greatly improve on the current peer review process. Full instructions can be found on Bench>Press http://submitoem.bmijournals.com and OEM online at http://www.occenvmed.com. Please contact Natalie Davies, Project Manager, ndavies@bmigroup.com.

Anne Cockcroft

Editor, OEM 